\title{
Os atuais desafios da Atenção Domiciliar na Atenção Primária à Saúde: uma análise na perspectiva do Sistema Único de Saúde
}

\author{
The current challenges of Home Care in Primary Health Care: an analysis in the National Health \\ System perspective
}

\section{Los desafíos actuales de la atención a domicilio en la atención primaria a salud: un análisis desde la perspectiva del Sistema Nacional de Salud}

\begin{abstract}
Leonardo Cançado Monteiro Savassi. Universidade Federal de Ouro Preto (UFOP); Universidade Federal de Minas Gerais (UFMG); Programa Multicêntrico de Qualificação em Atenção Domiciliar a Distância, da Universidade Aberta do SUS (UNASUS); Sociedade Brasileira de Medicina de Família e Comunidade (SBMFC). Ouro Preto, MG, Brasil. E-mail: leosavassi@gmail.com (Autor correspondente)
\end{abstract}

\section{Resumo}

A Política Nacional de Atenção Domiciliar (PNAD) definiu três níveis de atenção domiciliar (AD), reconhecendo o primeiro nível (AD1) como responsabilidade das equipes de Atenção Primária à Saúde (APS). Ao longo da história, a legislação passou por alterações importantes e essa política representa a valorização dos serviços de APS como âmbito privilegiado do cuidado, sendo responsável pela maior parte dos cuidados em AD no Brasil. O cuidado no domicílio deve ser feito sob critérios de elegibilidade específicos, e um planejamento adequado. A visita domiciliar (VD) é um momento ímpar para o conhecimento do contexto da pessoa sob AD e deve se pautar pela observação ativa, pela abordagem da família e reconhecimento dos determinantes sociais presentes. Assim, é necessário que o profissional de saúde visitador tenha um rol de competências profissionais bem definidas, que em geral não são contempladas na graduação dos cursos de saúde, e de maneira insuficiente pelos cursos de pós-graduação. A PNAD representou um avanço ao reconhecer a APS como responsável pela AD1 numa lógica de cuidados contínuos crescentes, mas as lacunas no entendimento entre equipes de AD e APS, de formação profissional, e de sobrecarga de tarefas persistem. Este estudo apresenta uma análise crítica da implantação da PNAD até o momento, baseada na legislação vigente confrontada a prática das equipes de APS trabalhando no Sistema Único de Saúde.

\begin{abstract}
The National Home Care Policy (NHCP) has defined three levels of home care $(\mathrm{HC})$, recognizing the first level (HC1) as responsibility of Primary Health Care (PHC) teams. Throughout history, the legislation has gone through major changes and this policy is the enhancement of primary care services as a privileged source of care, accounting for most of the $\mathrm{HC}$ in Brazil. The HC should be done under specific eligibility criteria, and proper planning. The Home Visit (HV) is a unique moment for the knowledge of the context of the person under $\mathrm{HC}$ and should be guided by active observation, family approach and recognition of social determinants. It is therefore necessary the visiting health professional to have a roll of well-defined professional skills, which are generally not included in undergraduate health courses, and insufficient in graduate courses. The $\mathrm{NHCP}$ represented a step forward in recognizing the $\mathrm{PHC}$ as responsible for $\mathrm{HC} 1 \mathrm{in}$ an increasing continuing care sense, but the gaps in the relationship between $\mathrm{HC}$ and $\mathrm{PHC}$ teams, the insufficient vocational training, and overhead tasks remain. This study presents a critical analysis of the implementation of the NHCP to date, based on current legislation confronted to the practice of PHC teams working in the Unified Health System.
\end{abstract}

Como citar: Savassi LCM. Os atuais desafios da Atenção Domiciliar na Atenção Primária à Saúde: uma análise na perspectiva do Sistema Único de Saúde. Rev Bras Med Fam Comunidade. 2016;11(38):1-12. http://dx.doi.org/10.5712/rbmfc11(38)1259
Palavras-chave: Atenção Primária à Saúde Serviços de Assistência Domiciliar Sistema Único de Saúde Saúde da Família

\section{Keywords:}

Primary Health Care Home Care Services Brazilian Unified Health System Family Health

Fonte de financiamento: declara não haver. Parecer CEP: não se aplica. Conflito de interesses: declara não haver. Procedência e revisão por pares: revisado por pares. Recebido em: 30/12/2015. Aprovado em: 25/06/2016. 


\section{Resumen}

La Política Nacional de Atención Domiciliaria (PNAD) ha definido tres niveles de Atención Domiciliaria (AD), reconociendo el primer nivel (AD1) como la responsabilidad de los equipos de Atención Primaria de Salud (APS). A lo largo de la historia, la legislación ha pasado por grandes cambios y esta política es lo reconocimiento de los servicios de atención primaria como una fuente privilegiada de la atención, que representa la mayor parte de la AD en Brasil. La AD debe hacerse bajo criterios de elegibilidad específicos y una planificación adecuada. La visita domiciliaria (VD) es un momento único para el conocimiento del contexto de la persona en $A D$ y debe guiarse por la observación activa, enfoque familiar y el reconocimiento de los determinantes sociales. Así, es necesario que lo profesional de salud visitador tenga una lista de habilidades profesionales bien definidos, que generalmente no se incluyen en los cursos de pregrado de salud y son insuficientes en los cursos de posgrado. EI PNAD representó un paso adelante en el reconocimiento de la APS como responsable de AD1 en sentido de una atención continua creciente, pero las diferencias en la relación entre los equipos de AD y APS, la formación profesional insuficiente, y las tareas generales demasiadas perseveran. Este estudio presenta un análisis crítico de la aplicación de la NPHC hasta la fecha, basada en la legislación vigente frente a la práctica de los equipos de APS que trabajan en el Sistema Único de Salud.

\section{INTRODUÇÃO}

A Política Nacional de Atenção Domiciliar define a atenção domiciliar (AD) no Sistema Único de Saúde como uma "modalidade de atenção à saúde substitutiva ou complementar às já existentes, caracterizada por um conjunto de ações de promoção à saúde, prevenção e tratamento de doenças e reabilitação prestadas em domicílio, com garantia de continuidade de cuidados e integrada às redes de atenção à saúde". 1

O cuidado no domicílio é uma das tarefas das equipes de Atenção Primária à Saúde (APS), em especial as equipes de saúde da família (eSF), que desde sua regulamentação têm entre suas atribuições executar ações de assistência na unidade básica de saúde, no domicílio e na comunidade. ${ }^{2}$

Parte importante do cuidado em saúde por estas equipes deve ser feita intradomicílios, entendendo a visita como uma ferramenta de acesso, de integralidade e do cuidado ao longo do tempo, que guarda relação íntima com a própria APS como nível de cuidados mais próximo do cidadão e, portanto, capaz de se moldar de modo mais flexível às suas necessidades. ${ }^{3}$ Para que as visitas domiciliares (VD) sejam efetivas, são necessários critérios de inclusão de pacientes de maneira clara e efetiva, para evitar tanto o dispêndio desnecessário de tempo quanto a negligência de situações que exigem o cuidado no domicílio.,4

Porém, uma ferramenta capaz de organizar plenamente o cuidado no lar dos usuários ainda não foi desenvolvida pelos serviços, sendo este um enorme desafio no cotidiano dos Serviços de Atenção Domiciliar (SAD) e de APS. Alguns estudos procuraram estabelecer critérios, pautados pela vulnerabilidade familiar ou pela clínica individual, mas que ainda não estão claramente sistematizados. Embora a individualização de cada caso seja desejável, parâmetros globais são relevantes para organizar o processo de trabalho destas equipes, em especial para o planejamento de ações em saúde, sendo os casos controversos analisados a partir de exceções ao processo. ${ }^{4-8}$

As VD representam de $10,1 \%$ a $11,5 \%$ de todos os contatos entre médicos de família e seus pacientes, respectivamente na Inglaterra e no Canadá, países com Atenção Primária bem estruturada. ${ }^{9,10}$ No Brasil, médicos dedicam até sete dias e enfermeiras até oito dias do mês para visitas. Enfermeiras fazem 5,76 $\mathrm{VD} /$ semana e médicos $3,74 \mathrm{VD} /$ semana, e a média de tempo dedicado a visitas é de $11,86 \%$ da carga horária semanal, sendo pior para médicos, com menos de $10 \%$ do tempo dedicado à VD. ${ }^{11,12}$

Considerando a casa como o espaço de maior intimidade da pessoa, é preciso ter claro que, enquanto no hospital o paciente está em território médico com regras rígidas, no domicílio são os profissionais de 
saúde que adentram o território do outro. Isto se reflete em uma exigência de um perfil de atuação específico, com habilidades, conhecimentos e atitudes diferenciados para a prática neste âmbito de cuidados. ${ }^{4,5,8}$

Assim, a adoção de uma nova Política Nacional de Atenção Domiciliar pelo Ministério da Saúde (MS) implica em novos arranjos organizacionais no território e em novos desafios para a organização das redes de atenção à saúde. São novas tecnologias de cuidados somadas àquelas já existentes, que em última análise modificam as demandas na formação de profissionais de saúde aptos para exercer suas funções nesse âmbito. ${ }^{1}$

\section{MÉTODO}

Trata-se de investigação exploratória pautada pela revisão da legislação vigente sobre políticas públicas atinentes à organização da $A D$ no Sistema Único de Saúde, seguida da análise crítica e reflexiva das atuais políticas frente aos cenários reais de prática de APS sob a ótica do autor. O objetivo deste ensaio é discutir os conceitos, a legislação pertinente e as competências dos profissionais envolvidos, sob a perspectiva das equipes de APS, responsáveis pela $A D$ de nível 1, que representa o primeiro nível de atenção às pessoas impossibilitadas a comparecer a serviços de saúde, conforme veremos a seguir.

\section{RESULTADOS}

\section{Evolução da Atenção Domiciliar no Brasil}

Até o século XIX, o poder público foi ausente em termos de políticas públicas, cabendo a entidades religiosas as poucas ações neste campo. A primeira instituição responsável por qualquer ação coletiva na área da saúde foi a Diretoria Geral de Saúde Pública (DGSP), criada em 1897 e subordinada ao Ministério da Justiça e Negócios Interiores. ${ }^{13}$

Nos primórdios do último século, a Medicina era privada, e os médicos de família eram os profissionais que atendiam clientes abastados eminentemente em casa, enquanto a população comum era cuidada por entidades filantrópicas, como as Santas Casas, pela medicina caseira ou mesmo pelo curandeirismo. Mas ao longo do século, em todo o mundo, as VD foram declinando como fonte do cuidado, sendo progressivamente substituídas pelos serviços ambulatoriais, pelos hospitais e por consultórios particulares. . $^{914,15}$

No Brasil, as VD começaram a ser feitas inicialmente pelos agentes sanitários para detectar focos de doenças, porém com uma perspectiva de vigilância a saúde e punição, como foi o caso da Hanseníase, em especial após a centralização estatal promovida por Getúlio Vargas. ${ }^{13-16}$

Os governos posteriores seguiram a perspectiva de um sistema de saúde Bismarckiano, regido por seguros sociais de saúde ligados ao trabalho e limitado papel governamental, e a Medicina chegou a seu ápice de superespecialização dos serviços médicos, iniciada em 1910 com Flexner, que elevou o hospital ao papel de principal responsável pelo cuidado, à custa da fragmentação do cuidado e da redução do papel da APS. Declinaram concomitantemente, portanto, os cuidados realizados no domicílio, que foram assumidos pelas instituições hospitalares. ${ }^{16,17}$

A primeira experiência Brasileira de AD ocorreu em 1949, com o Serviço de Assistência Médica Domiciliar de Urgência (SAMDU), vinculado ao Ministério do Trabalho, sendo a primeira ação de atendimento 
domiciliar brasileira organizada como um serviço. A atenção domiciliar foi iniciada como uma atividade planejada pelo setor público com o Serviço de Assistência Domiciliar do Hospital de Servidores Públicos do Estado de São Paulo (HSPE). ${ }^{16}$

A partir de 1978, impulsionado pelas discussões da Conferência de Alma-Ata, ${ }^{18}$ o movimento sanitário se reorganizaria, sendo realizada a VIII Conferência Nacional de Saúde em 1986, que consolidaria as propostas da Reforma Sanitária Brasileira no sentido de um modelo Beveridgeano, de sistema nacional de saúde, resultando na Constituição Federal de 1988, que definiu o Sistema Único de Saúde (SUS) brasileiro.

Neste meio tempo, começam a se desenvolver alguns serviços de apoio a hospitais e surgem as primeiras experiências de Serviços de Atenção Domiciliar, impulsionadas por iniciativa ligadas a Secretarias Municipais de Saúde, ou a hospitais públicos. ${ }^{16}$

Com a progressiva implantação do SUS, surge o Programa dos Agentes Comunitários de Saúde (ACS), agentes visitadores responsáveis por estabelecer elos entre o serviço de saúde e a população, e posteriormente o Programa Saúde da Família, com foco na atenção à saúde sob as vertentes da prevenção, promoção, tratamento, cura e reabilitação. ${ }^{2}$

A APS se reorganizava, então, sob um novo contexto com outras possibilidades de intervenção, e os profissionais médicos e enfermeiros voltam a lançar mão da VD como uma ferramenta de assistência, desta vez com foco na população menos favorecida.

A legislação relativa aos cuidados no domicílio se ampliou a partir daí: a Portaria 2.416/1998 estabeleceria requisitos para credenciamento de hospitais e critérios para realização de internação domiciliar no SUS, a cargo destas instituições. A seguir, a Portaria GM/MS nำ1.531/2001 regulamentou a ventilação mecânica não invasiva em domicílio aos pacientes portadores de distrofia muscular progressiva, sob os cuidados de equipes específicas (e não da APS) para tal, financiadas pelo SUS. ${ }^{16}$

No ano seguinte, a Lei 10.424/2002 regulamentaria a assistência domiciliar no SUS e a Portaria SAS/MS 249/2002 estabeleceria a assistência domiciliar como modalidade assistencial a ser desenvolvida na Assistência à Saúde do Idoso; por fim, a Portaria GM/MS 1.370/2008, regulamentada pela Portaria SAS/MS 370/2008, ampliava o rol de doenças elegíveis para cadastramento em oxigenoterapia domiciliar, todas estas ações fora do escopo da APS. ${ }^{16}$

A evolução da atenção no domicílio no âmbito da APS e dos SAD passaria a se dar de maneira concorrente e não integrada, com limites sobrepostos entre os serviços. A visão de alguns autores, e do próprio MS, era de serviços distintos pertencentes a níveis de atenção diferentes e, portanto, sem intercooperação. ${ }^{19,20}$

Ainda com este viés, a Portaria Ministerial 2529/2006, e a Resolução no 11 da Diretoria Colegiada da Agência Nacional de Vigilância Sanitária (ANVISA), de 26 de janeiro de 2006, vislumbrariam os serviços como estruturas de níveis e organizações diversas, não integradas. Ao mesmo tempo, a Política Nacional de Atenção Básica (PNAB) de 2006 definia a VD e a AD dentro do processo de trabalho das equipes de APS. ${ }^{21}$ Persistia, portanto, uma atenção sobreposta entre os SAD e a APS, não sinérgica e com retrabalho. É importante ressaltar que a legislação sobre AD em serviços suplementares e privados ainda é regida pela RDC no 11/2006 da ANVISA. ${ }^{22}$

Em 2011, o MS redefine a AD no âmbito do SUS e pela primeira vez enxerga a APS e os SAD como elementos integrados de cuidados, propondo níveis de complexidade clínica e tecnológica que delimitem 
o cuidado entre eles, reconhecendo as ações domiciliares da APS como o nível 1 da Atenção Domiciliar (AD1), que se integram a dois níveis de $A D$ (AD2 e AD3) realizados por Equipes Multiprofissionais de $A D$ (EMAD) destinadas a este fim, sob a necessidade de integração entre níveis (Quadro 1). ${ }^{1,4}$

Quadro 1. Modalidades de Atenção Domiciliar e seus critérios de inclusão.

\begin{tabular}{|c|c|c|}
\hline Modalidade & A quem se destina & Quem realiza \\
\hline AD 1 & $\begin{array}{l}\text { Usuários que: } \\
\text { I - possuam problemas de saúde controlados/compensados } \\
\text { e com dificuldade ou impossibilidade física de locomoção } \\
\text { até uma unidade de saúde; } \\
\text { II - necessitem de cuidados de menor complexidade, } \\
\text { incluídos os de recuperação nutricional, de menor } \\
\text { frequência, com menor necessidade de recursos de saúde } \\
\text { e dentro da capacidade de atendimento das Unidades } \\
\text { Básicas de Saúde (UBS); e } \\
\text { III - não se enquadrem nos critérios previstos para as } \\
\text { modalidades AD2 e AD3 descritos nesta Portaria. }\end{array}$ & $\begin{array}{l}\text { A AD1 é de responsabilidade das equipes de APS, por } \\
\text { meio de VD regulares, no mínimo, uma vez por mês, } \\
\text { apoiadas pelos NASF, ambulatórios de especialidades e } \\
\text { de reabilitação. }\end{array}$ \\
\hline AD 2 & $\begin{array}{l}\text { Usuários com problemas de saúde + dificuldade ou } \\
\text { impossibilidade física de locomoção até uma UBS } \\
\text { com maior frequência de cuidado, recursos de saúde e } \\
\text { acompanhamento contínuo, definidas em um rol amplo de } \\
\text { procedimentos domiciliares. }\end{array}$ & $\begin{array}{l}\text { A AD2 é exercida pelas equipes multiprofissionais de } \\
\text { atenção domiciliar (EMAD) apoiadas pelas equipes } \\
\text { multiprofissionais de apoio (EMAP) }\end{array}$ \\
\hline AD 3 & $\begin{array}{l}\text { Usuários com problemas de saúde + dificuldade ou } \\
\text { impossibilidade física de locomoção até uma UBS } \\
\text { com maior frequência de cuidado, recursos de saúde e } \\
\text { acompanhamento contínuo e uso de equipamentos e } \\
\text { os seguintes procedimentos: oxigenoterapia, ventilação } \\
\text { mecânica não invasiva, diálise peritoneal e paracentese. }\end{array}$ & $\begin{array}{l}\text { A AD3 também é exercida pelas equipes multiprofissionais } \\
\text { de atenção domiciliar (EMAD) apoiadas pelas equipes } \\
\text { multiprofissionais de apoio (EMAP) }\end{array}$ \\
\hline
\end{tabular}

Fonte: Ministério da Saúde, $2016 .^{4}$

O papel das EMAD não substitui as ações realizadas no domicílio pelas equipes de APS, em especial as eSF. Pelo contrário, ao considerar a AD1 como de responsabilidade destas equipes, o MS reconhece o seu trabalho como um eixo fundamental ao mesmo tempo em que as EMAD se apresentam como uma possibilidade de serem complementares, quando as demandas dos pacientes superam a capacidade de resposta das equipes de APS.

A PNAB de 2011 reforçaria esta integração, e a AD1 passaria a fazer parte do rol de atividades das equipes de APS inclusive em seu sistema de informação: o Sistema de Informações da Atenção Básica (SIS-AB), ligado ao e-SUS, contempla a classificação da complexidade do cuidado domiciliar no do rol de ações das equipes de APS. ${ }^{23}$

\section{A Atenção Domiciliar de Nível 1 e a Visita Domiciliar na APS}

A VD é considerada uma ferramenta importante e uma ação aprazível para profissionais de saúde, em especial aqueles que atuam no âmbito da APS, mas no cotidiano das equipes trata-se de uma atividade realizada em proporção aquém do esperado. ${ }^{3}$

De acordo com a PNAD, a grande maioria das pessoas estará sob cobertura exclusivamente por equipes que praticam a AD1, ou seja, equipes de atenção básica (eAB) e de Saúde da Família (eSF), sendo exatamente aquelas pessoas que geralmente não contam com a infraestrutura de saúde dos outros níveis de atenção no território. Os critérios de inclusão para AD2 e AD3 também apontam que a grande 
maioria das pessoas sob AD, mesmo em municípios com EMAD e EMAP, serão aquelas com problemas crônicos de saúde ou com critérios de inclusão para AD1. Assim, a maior parte da AD no sistema público será exercido pela APS. ${ }^{1,4,24}$

Algumas dificuldades são apontadas na integração entre as equipes que praticam AD1 e as EMAD e EMAP, no que se refere aos fluxos de inclusão e exclusão nestes serviços, sendo este um exercício ainda incompleto da referência e contrarreferência. E, ainda que as EMAD assumam o cuidado domiciliar de pacientes em AD2 e AD3, a responsabilidade pela coordenação e longitudinalidade do cuidado continua sendo das equipes de APS, pois esses pacientes não deixam de estar sob o encargo de suas respectivas equipes em seu território. ${ }^{16,24}$

Em especial nas eSF, em um cenário de cobertura mínima de 2.400 pessoas e teto - muitas vezes ultrapassado - de 4.000 usuários, há no planejamento pouco tempo disponível para que os profissionais de saúde a realizem com uma frequência capaz de suprir as demandas da comunidade adscrita.

As visitas podem ser classificadas em VD meio - como instrumento de conhecimento do contexto próximo familiar - e VD fim - com fins específicos de atuação pré-determinados. ${ }^{5}$ São ainda divididas em quatro grandes grupos: VD para doenças, VD para pacientes terminais, VD para avaliação do contexto e VD de acompanhamento de pós alta hospitalar. ${ }^{3}$

A reunião de equipe é o maior motivador para a realização de visitas, sendo o momento no qual a equipe é capaz de discutir aqueles casos que deveriam ser priorizados, bem como qual seria o profissional mais adequado para tal. ${ }^{11}$

Uma VD inicia-se no planejamento: a partir da demanda de um ACS nas equipes em que ele está presente, de uma necessidade relatada no Acolhimento a Demanda Espontânea ou por meio de outros contatos da pessoa (ou seus familiares) com o serviço, identifica-se a necessidade de dedicar um espaço na agenda semanal para abordar a situação específica.

As equipes de APS, sejam eAB ou eSF, geralmente se planejam para realizar VD pautadas por situações clínicas individuais, ficando em um segundo plano aquelas visitas que visam abordar a dinâmica e funcionalidade familiar, ou endereçar ações à vulnerabilidade social ou clínica da família como um todo.

Uma vez identificada uma demanda e agendada a visita, é o momento de planejar as etapas burocráticas da ação, que envolvem a definição: a) do horário de visita, combinando a disponibilidade dos profissionais envolvidos na visita (motorista, ACS, auxiliar de enfermagem, médico, enfermeiro, dentista) com as possibilidades da família; b) o meio de transporte, caso haja veículo disponível e também de acordo com a disponibilidade do mesmo, ou carro próprio; c) o material necessário para a visita, incluindo caixa de medicamentos e possibilidade de exames rápidos como glicemia capilar; d) o itinerário, considerando que os profissionais, em geral, determinam um turno por semana para ações no território. ${ }^{24}$

Algumas pactuações são necessárias para que se processe da melhor maneira o cuidado no domicílio. A Associação Mineira de Medicina de Família e Comunidade (AMMFC) propõe cinco passos para se programar uma VD e a partir daí avaliar o processo de cuidado em AD1 (Quadro 2). ${ }^{24}$

Ao contrário dos SAD, quando a ausência de cuidador ou de infraestrutura impedem a inclusão do paciente nas modalidades AD2 e AD3, no caso da AD1, pela vinculação territorial, o paciente continua sob a responsabilidade das eAB/eSF. A ausência destes elementos implicará na construção de redes sociais de apoio e da intersetorialidade para fins de atender às necessidades de cada família em seu contexto particular. 
Quadro 2. Cinco passos para definir o cuidado domiciliar.

1. Avaliação da resolutividade da VD (A VD será resolutiva);

2. Avaliação da razoabilidade da VD (A VD é a melhor alternativa);

3. Aderência do usuário e sua família ao acompanhamento (engajamento e corresponsabilização);

4. Autorização do usuário e da família (termo de consentimento que deverá ser anexado ao prontuário);

5. Análise da infraestrutura domiciliar (avaliação para análise de caso, classificação da complexidade e determinação do plano de cuidados).

Fonte: AMMFC apud Savassi \& Cunha, $2016^{24}$

Definida a viabilidade, o momento da visita é envolto por um ambiente peculiar ao se acessar o âmbito mais íntimo do paciente. Isto exige um ritual próprio cercado de simbologias e consequentes tarefas, tais como: ${ }^{5}$

- Legitimar o papel do ACS nas equipes de APS em que se faz presente, deixando-o encabeçar a entrada no domicílio;

- Verificar previamente se cães ou outros animais domésticos não oferecem riscos para a equipe;

- Solicitar formalmente antes de adentrar o recinto: "Com licença, posso entrar?"

- Determinar o melhor local para a anamnese, avaliando a necessidade de respeitar a privacidade do paciente.

- Conduzir uma anamnese que apresenta vários sujeitos (minimamente os familiares presentes e os membros da equipe), portanto, vários canais paralelos de comunicação.

- Realizar o exame físico e os procedimentos necessários superando as barreiras do domicílio;

- Ao final da consulta, estabelecer encaminhamentos claros e pactuar papéis dos atores envolvidos.

A VD é um momento ideal para conhecer o contexto familiar e social e com isto entender de maneira mais completa o adoecimento daquela pessoa e não apenas os aspectos biológicos da doença. Portanto, é pertinente que o profissional de saúde tenha um olhar para o ambiente interno, os riscos do domicílio, tanto aqueles sociais e comunitários como pestes, focos de doenças e condições sanitárias insalubres, quanto para aqueles internos como iluminação, ventilação, risco de quedas e acesso a condições mínimas de cidadania. ${ }^{25,26}$

É também o momento privilegiado para se identificar situações familiares importantes, tais como presença de cuidador, a liderança da família, as relações familiares de afeto e conflito, e com isto conseguir montar elementos que permitam entender como se dá a dinâmica familiar, com uso do genograma, ecomapa ou outros elementos de classificação e abordagem familiar.

Uma vez definida a necessidade de cuidados prolongados, o paciente se enquadrará na modalidade $A D 1$, e receberá cuidados frequentes domiciliares com menor complexidade tecnológica e menor exigência de tempo de cuidado pela equipe, conforme definido na Portaria 963/2013. ${ }^{4}$

A partir daí, desenha-se o projeto terapêutico para aquele paciente, composto pelo plano de cuidados, incluindo periodicidade e intensidade da atenção, e profissionais necessários para o cuidado por parte da equipe, bem como atribuições dos componentes familiares. ${ }^{8}$

\section{Competências do Profissional Visitador}

Partindo do preceito que a $A D$ apresenta um contexto peculiar de atuação, e que neste não há retaguarda do serviço de saúde, deve-se definir o campo de atuação profissional. Neste cenário, o profissional de saúde demanda competências específicas para o cuidado no domicílio, lançando mão de protagonismo e criatividade para estabelecer uma atuação eficaz. 
Por não atuar dentro da estrutura física e com instrumentos do serviço, a ação profissional no domicílio demandará algumas habilidades complementares que determinarão o perfil das competências deste indivíduo ao cuidar de pessoas restritas ao domicílio ou sob situações de disfuncionalidade ou de vulnerabilidade familiar.

Na prática da APS, o que se observa são profissionais médicos e enfermeiros aprendendo a lidar com estas situações experimentalmente no cenário de prática, sendo a atuação no serviço o momento de aprender a "cuidar em casa". O paciente domiciliar, então, se torna o objeto de aprendizagem deste profissional, de maneira não supervisionada.

A discussão sobre o perfil do profissional visitador tem-se ampliado, e diversos fóruns têm discutido em especial suas competências e formação. Assim, no ano de 2015 uma proposta de competências a serem cumpridas pelo profissional de saúde, em especial do médico, foi elaborada em um Simpósio ocorrido na Associação Paulista de Medicina, em colaboração com a Associação Paulista de Medicina de Família e Comunidade (APMFC): ${ }^{27}$

São habilidades esperadas do profissional visitador:

1. Protagonizar a tomada de decisões complexas;

2. Exercer a criatividade na prática de suas atividades clínicas;

3. Adaptar a melhor evidência científica para um contexto real;

4. Gerenciar conflitos familiares;

5. Incrementar a relação equipe-família;

6. Executar procedimentos com equipamentos mínimos e sem estrutura física ideal;

7. Atender independentemente do ciclo de vida das pessoas;

8. Classificar a vulnerabilidade e a funcionalidade familiar;

9. Abordar a família sob o ponto de vista social, clínico e sistêmico;

10. Reconhecer os elementos presentes na rede de apoio social;

11. Apoiar o cuidador do ponto de vista clínico e de aquisição de competências;

12. Classificar a complexidade do cuidado;

13. Lidar com situações de violência familiar e comunitária.

São atitudes esperadas do profissional visitador:

1. Empatia e disponibilidade para discutir situações fora do contexto da saúde.

2. Humildade ao adentrar o terreno do outro;

3. Respeito pelas crenças e modelos explicativos familiares do processo saúde-adoecimento;

4. Busca por consenso com a família na definição de metas de cuidado;

5. Discernimento para entender elementos antropológicos que interfiram no cuidado;

6. Observação ativa da família, do domicílio e da vizinhança;

7. Disponibilidade para ouvir e adaptar condutas à realidade local; 
8. Neutralidade em conflitos familiares, procurando estabelecer vias de diálogo;

9. Autoconhecimento para evitar que crenças pessoais ou sentimentos negativos em relação aos demais atores afetem a ação em saúde;

10. Vínculo com a família para se configurar como a fonte contínua de cuidados;

11. Desprendimento para abordar a morte e o luto de acordo com a crença de cada familiar;

12. Posicionamento claro diante de situações de violência familiar.

São conhecimentos esperados do profissional visitador:

1. A aplicação da melhor evidência clínica científica disponível para cada quadro clínico;

2. A técnica ideal de procedimentos comuns e suas possíveis adaptações;

3. O tratamento de situações pouco usuais na clínica ambulatorial;

4. A determinação social da saúde e suas vertentes;

5. Representação gráfica, classificação e abordagem familiar;

6. Gerenciamento de casos complexos e elaboração de planos de cuidados;

7. Instrumentos de avaliação de autonomia e mobilidade;

8. Instrumentos de avaliação de sobrecarga e abordagem do cuidador;

9. Formas de construção de redes de apoio social;

10. Noções de antropologia para entender a cultura local e transformar empecilhos em soluções;

11. Classificação da complexidade do cuidado;

12. Cuidados paliativos e a terminalidade da vida;

13. Óbito no domicílio e orientações funerárias;

14. Planejamento e gerenciamento de fluxos de atuação no domicílio.

Em suma, o rol de atividades dos profissionais de saúde que atuam em atenção domiciliar é denso e amplo. Este cenário torna-se ainda mais complexo quando os profissionais de saúde atuam em localidades com características específicas.

A atuação em regiões rurais remotas, por exemplo, amplia as necessidades de conhecimentos e habilidades dos profissionais para resolver situações específicas destes cenários ou que supram a ausência de referências de apoio. O mesmo é válido para populações ribeirinhas, onde uma proporção relevante de atendimentos se dará eminentemente no domicílio pela falta de acesso geográfico. ${ }^{28}$

Por outro lado, a atuação em periferias urbanas exige uma integração necessariamente mais coesa com a comunidade, pois a vulnerabilidade social somada à violência local exige que a equipe possa se adaptar aos limites impostos pelo modo de vida daquela população, exercendo o cuidado domiciliar sob os olhos do poder paralelo imposto pela falta de cidadania.

Como na graduação dos cursos da área da saúde não há uma carga horária extensa ou densa o suficiente para a formação destes profissionais, entende-se que uma formação específica destes profissionais é necessária, ao menos para o desenvolvimento das competências aqui elencadas. ${ }^{24}$ 
Além disto, o rol de competências esperadas, especialmente para os médicos, não é contemplada por uma única especialidade clínica, não havendo hoje no campo da formação desses profissionais nenhuma especialidade que abarque todos os conhecimentos necessários em seus respectivos programas de residência médica.

As residências em Medicina de Família e Comunidade e multiprofissional em Saúde da Família têm respondido parcialmente a estas demandas, e a discussão sobre a AD como cenário de prática em especial para Médicos de Família se amplia, porém, dadas as demandas de formação na área da AD, mesmo estas iniciativas não têm sido suficientes para uma abordagem integral do paciente em AD. ${ }^{27}$

A ampliação das habilidades e conhecimentos específicos para o cuidado no domicílio implica em um volume de competências e em um campo da prática que envolva todas as necessidades para o treinamento em serviço, que deveria intercalar a atuação nos campos da APS, hospital, unidades intensivistas e o próprio domicílio, com treinamento de habilidades específicas e conteúdo teórico voltado para os elementos clínicos mais prevalentes na AD.

\section{Conclusões}

A nova Política Nacional de Atenção Domiciliar representou um grande avanço ao reconhecer o cuidado domiciliar feito pelas equipes de APS no território como o primeiro nível da AD (AD1), roteirizando melhor as atribuições das equipes e sinalizando uma necessidade de complementaridade e continuidade do cuidado entre as equipes de APS e EMAD.

Entretanto, ainda persistem dificuldades por parte destas equipes em compartilhar o cuidado, em parte pela dificuldade de comunicação entre elas, em parte por um entendimento ainda insuficiente da política, muito nova em termos de tempo e de implantação de equipes.

Somam-se a isto as demandas de formação específica para $A D$, cujo conteúdo é pouco explorado durante a graduação. A ausência de uma formação adequada de pós-graduação para tais profissionais, no que diz respeito a habilidades e competências atitudinais, torna-se uma lacuna também não preenchida pela educação permanente.

Há um rico cenário de cuidados realizados nos domicílios pelas equipes de APS, especialmente eSF, em que a produção do cuidado é realizada com competência clínica e resultados relevantes pelos serviços, embora ainda pouco explorados pela pesquisa ou pelo ensino na graduação.

Trata-se do maior serviço de AD no Brasil, com cerca de 40 mil eSF, responsáveis pela parcela da população que não conta com EMAD por critérios populacionais. Além disso, mesmo em municípios com $S A D$, as equipes de APS são responsáveis pela maior parte dos cuidados realizados no domicílio, a saber, todos aqueles qualificados como AD1.

Assim, embora campo de prática da APS por 20 anos no Brasil, o cuidado no domicílio permanece negligenciado e ameaçado por uma demanda de cuidados que ultrapassa a capacidade de atendimento das equipes. Trata-se de um momento ímpar para uma rediscussão de suas potencialidades visando à formação adequada e a ampliação da rede de atenção à saúde no âmbito do SUS. 


\section{Referências}

1. Brasil. Ministério da Saúde. Portaria MS/GM no 2.527 de 27 de outubro de 2011. Redefine a atenção domiciliar no âmbito do Sistema Único de Saúde (SUS). Diário Oficial da União 208, de 28 out. Brasília: Ministério da Saúde; 2011.

2. Brasil. Ministério da Saúde. Portaria GM/MS no 1.886, de 18 de dezembro de 1997. Aprova normas e diretrizes do Programa de Agentes Comunitários de Saúde e do Programa de Saúde da Família. Diário Oficial da União 247, de 22 dez. Brasília: Ministério da Saúde; 1997. p.11-13.

3. Unwin BK, Jerant AF. The home visit. Am Fam Physician. 1999;60(5):1481-8. PMID: 10524492

4. Brasil. Ministério da Saúde. Portaria 825/2016. Redefine a atenção domiciliar no âmbito do Sistema Único de Saúde (SUS) e atualiza as equipes habilitadas. Diário Oficial da União 78, de 26 abr. Brasília: Ministério da Saúde; 2016. p.33-8.

5. Coelho FLG, Savassi LCM. Aplicação da escala de risco familiar como instrumento de priorização de visitas domiciliares. Rev Bras Med Fam Comunidade. 2004;1(2):19-26. DOI: http://dx.doi.org/10.5712/rbmfc1(2)104

6. Escola de Saúde Pública do Estado de Minas Gerais. Implantação do Plano Diretor da Atenção Primária à Saúde. Saúde em Casa. Oficina 3. Guia do participante [internet]. Belo Horizonte; 2008. [Acesso 2015 Abr 08]. Disponível em: http://www. esp.mg.gov.br/wp-content/uploads/2009/04/pdaps-oficina3-participante.pdf

7. Nascimento FG, Prado TN, Galavote HS, Maciel PA, Lima RCD, Maciel ELN. Aplicabilidade de uma escala de risco para organização do processo de trabalho com famílias atendidas na Unidade Saúde da Família em Vitória (ES). Ciênc Saúde Coletiva. 2010;15(5):2465-72. DOI: http://dx.doi.org/10.1590/S1413-81232010000500021

8. Savassi LCM, Carvalho HRO, Mariano FM, Lamberti CA, Mendonça MF, Yamana GF, et al. Proposta de um protocolo de classificação de risco para atendimento domiciliar individual na atenção primária à saúde. J Manag Prim Health Care. 2012;3(2):151-7. DOI: http://dx.doi.org/10.1234/jmphc3/jmphc

9. Aylin P, Majeed FA, Cook DG. Home visiting by general practitioners in England and Wales. BMJ. 1996;313(7051):207-10. DOI: http://dx.doi.org/10.1136/bmj.313.7064.1085

10. Bergeron R, Laberge A, Vézina L, Aubin M. Which physicians make home visits and why? A survey. CMAJ. 1999;161(4):36973. PMID: 10478159

11. Peres EM, Andrade AM, Dal Poz MR, Grande NR. The practice of physicians and nurses in the Brazilian Family Health Programme - evidences of change in the delivery health care model. Hum Resour Health. 2006;4:25. DOI: http://dx.doi. org/10.1186/1478-4491-4-25

12. Savassi LCM, Savassi FM, Gomes ALFM, De-Paula HGM, Souza RA. Avaliação da ferramenta visita domiciliar por profissionais da ESF. 10o Congresso Brasileiro de Medicina de Família e Comunidade. Florianópolis; 2009.

13. Ducatti I. A hanseníase no Brasil na era Vargas e a profilaxia do isolamento compulsório: estudos sobre o discurso científico legitimador [Dissertação de mestrado]. São Paulo: Departamento de História, Programa de Pós-Graduação em História Social, Universidade de São Paulo; 2009. 199p.

14. Savassi LCM, Dias MF. Grupos de estudo em saúde da família. Módulo Visita domiciliar. [internet] 2006. [Acesso 2012 Abr 07]. Disponível em: http://www.smmfc.org.br/gesf/gesfvd.htm

15. Meyer GS, Gibbons RV. House calls to the elderly--a vanishing practice among physicians. N Engl J Med. 1997;337(25):181520. DOI: http://dx.doi.org/10.1056/NEJM199712183372507

16. Brasil. Ministério da Saúde. Secretaria de Atenção à Saúde. Departamento de Atenção Básica. Caderno de Atenção Domiciliar. Brasília: Ministério da Saúde; 2012.

17. Aguiar RAT. A construção internacional do conceito de Atenção Primária à Saúde (APS) e sua influência na emergência e consolidação do Sistema Único de Saúde no Brasil [Dissertação de mestrado]. Belo Horizonte: Universidade Federal de Minas Gerais; 2003.

18. Declaração de Alma-Ata. Conferência Internacional sobre cuidados primários de saúde; 6-12 de setembro 1978; Alma-Ata; USSR. In: Ministério da Saúde (BR). Secretaria de Políticas de Saúde. Projeto Promoção da Saúde. Declaração de AlmaAta; Carta de Ottawa; Declaração de Adelaide; Declaração de Sundsvall; Declaração de Santafé de Bogotá; Declaração de Jacarta; Rede de Megapaíses; Declaração do México. Brasília (DF): Ministério da Saúde; 2001. p. 15. 
19. Brasil. Ministério da Saúde. Grupo Hospitalar Conceição. Manual de assistência domiciliar na atenção primária à saúde. Porto Alegre; 2003.

20. Brasil. Ministério da Saúde. Secretaria de Atenção à Saúde. Departamento de Atenção Básica. Política nacional de atenção básica. Brasília: Ministério da Saúde; 2006. p.60.

21. Brasil. Ministério da Saúde. Portaria n.o 2.529 de 19 de Outubro de 2006. Institui a Internação Domiciliar no âmbito do SUS. Diário Oficial da União 202, de 20 out 2006. Brasília: Ministério da Saúde; 2006. p.145-8.

22. Brasil. Ministério da Saúde. Resolução da Diretoria Colegiada da Agência Nacional de Vigilância Sanitária (RDC/ANVISA) no 11, de 26 de janeiro de 2006. Diário Oficial da União Suplemento ao No - 21. Brasília: Ministério da Saúde; 2006. p.1-2.

23. Brasil. Ministério da Saúde. Secretaria de Atenção à Saúde. Departamento de Atenção Básica. Política Nacional de Atenção Básica/Ministério da Saúde. Secretaria de Atenção à Saúde. Departamento de Atenção Básica. Brasília: Ministério da Saúde; 2012.

24. Savassi LCM, Cunha CL. Atenção Domiciliária como Estratégia do Cuidado na Atenção Primária à Saúde. In: Cunha CL, Leoneza I. Guia de Trabalho para o Enfermeiro na Atenção Primária à Saúde. Rio de Janeiro: Rubio; 2016.

25. Mendes AO, Oliveira FA. Visitas domiciliares pela equipe de Saúde da Família: reflexões para um olhar ampliado do profissional. Rev Bras Med Fam Comunidade. 2007;2(8):253-60. DOI: http://dx.doi.org/10.5712/rbmfc2(8)64

26. McWhinney IR, Freeman T. Textbook of family medicine. 3nd ed. New York: Oxford University Press; 2009. p.472.

27. Savassi LCM. Formação médica em Atenção Domiciliar. In: I Jornada de Medicina de Família e Comunidade e Atenção Domiciliar. São Paulo: Associação Paulista de Medicina. [internet] 2014. [Acesso 2016 Abr 23]. Disponível em: https:// sites.google.com/site/leosavassi

28. Humphreys RC. Home visiting in a rural practice. Br Med J (Clin Res Ed). 1981;282(6258):115-6. DOI: http://dx.doi. org/10.1136/bmj.282.6262.483-c 